Pacific Journal of Mathematic 


\title{
REGULAR SEMIGROUPS WHOSE IDEMPOTENTS SATISFY PERMUTATION IDENTITIES
}

\author{
MIYUKI YAMADA
}

This paper is concerned with a certain class of regular semigroups. It is well-known that a regular semigroup in which the set of idempotents satisfies commutativity $x_{1} x_{2}=$ $x_{2} x_{1}$ is an inverse semigroup firstly introduced by V. V. Vagner, and the structure of inverse semigroups was clarified by $A$. $E$. Liber, W. D. Munn, G. B. Preston and V. V. Vagner, etc. By a generalized inverse semigroup is meant a regular semigroup in which the set of idempotents satisfies a permutation identity $x_{1} x_{2} \cdots x_{n}=x_{p_{1}} x_{p_{2}} \cdots x_{p_{n}}$ (where $\left(p_{1}, p_{2}, \cdots, p_{n}\right)$ is a nontrivial permutation of $(1,2, \cdots, n))$. $N$. Kimura and the author proved in a previous paper that any band $B$ satisfying a permutation identity satisfies normality $x_{1} x_{2} x_{3} x_{4}=x_{1} x_{3} x_{2} x_{4}$. Such a $B$ is called a normal band, and the structure of normal bands was completely determined. In this paper, first a structure theorem for generalized inverse semigroups is established. Next, as a special case, it is proved that a regular semigroup is isomorphic to the spined product (a special subdirect product) of a normal band and a commutative regular semigroup if and only if it satisfies a permutation identity. The problem of classifying all permutation identities on regular semigroups into equivalence classes is also solved. Finally, some theorems are given to clarify the mutual relations between several conditions on semigroups. In particular, it is proved that an inverse semigroup satisfying a permutation identity is necessarily commutative.

A semigroup $S$ is called regular if it satisfies the following:

(1.1) For any element $a$ of $S$, there exists an element $a^{*}$ such that $a a^{*} a=a$.

A semigroup $G$ admitting relative inverses introduced by Clifford [1], i.e., a semigroup $G$ satisfying the following condition (1.2) is clearly regular:

(1.2) For any element $a$ of $G$, there exists an element $a^{*}$ such that $a^{*} a=a a^{*}$ and $a a^{*} a=a$.

However, the converse is not true. It is well-known that a semigroup is a semigroup admitting relative inverses if and only if it is a union of groups. Consider the symmetric inverse semigroup on the set $\{1,2\}$ (for definition, see [3], p. 29). Then this semigroup is regular but not a union of groups. 
Next, we define a (polynomial) identity as follows: Let $X=$ $\left\{x_{1}, x_{2}, \cdots, x_{n}\right\}$ be a set in which each element $x_{i}$ is called a variable. Let $W_{1}\left(x_{1}, x_{2}, \cdots, x_{n}\right)$ and $W_{2}\left(x_{1}, x_{2}, \cdots, x_{n}\right)$ be two words consisting of elements of $X$ (each of $W_{1}\left(x_{1}, x_{2}, \cdots, x_{n}\right)$ and $W_{2}\left(x_{1}, x_{2}, \cdots, x_{n}\right)$ need not contain all letters $\left.x_{1}, x_{2}, \cdots, x_{n}\right)$. Then the pair of the two words $W_{1}\left(x_{1}, x_{2}, \cdots, x_{n}\right)$ and $W_{2}\left(x_{1}, x_{2}, \cdots, x_{n}\right)$ is called an identity in the variables $x_{1}, x_{2}, \cdots, x_{n}$ and is usually written in the form

$$
W_{1}\left(x_{1}, x_{2}, \cdots, x_{n}\right)=W_{2}\left(x_{1}, x_{2}, \cdots, x_{n}\right) .
$$

By a permutation identity in the variables $x_{1}, x_{2}, \cdots, x_{n}$, we shall mean an identity

$$
x_{1} x_{2} \cdots x_{n}=x_{p_{1}} x_{p_{2}} \cdots x_{p_{n}},
$$

where $\left(p_{1}, p_{2}, \cdots, p_{n}\right)$ is a nontrivial permutation of $(1,2, \cdots, n)$.

For example, the identities

(C) commutativity $x_{1} x_{2}=x_{2} x_{1} ;(L . N)$ left normality $x_{1} x_{2} x_{3}=x_{1} x_{3} x_{2}$; $(R . N)$ right normality $x_{1} x_{2} x_{3}=x_{2} x_{1} x_{3}$; and $(N)$ normality $x_{1} x_{2} x_{3} x_{4}=$ $x_{1} x_{3} x_{2} x_{4}$

are all permutation identities, while each of the identities

(L.S) left singularity $x_{1} x_{2}=x_{1} ;(R . S)$ right singularity $x_{1} x_{2}=x_{2}$; and $(R)$ rectangularity $x_{1} x_{2} x_{3}=x_{1} x_{3}$

is not a permutation identity.

If a subset $M$ of a semigroup $G$ satisfies the following condition (1.5), then we shall say that $M$ satisfies the identity (1.3) (in $G$ ):

(1.5) For any mapping $\varphi$ of $X$ into $M$, the equality $W_{1}\left(\varphi\left(x_{1}\right), \varphi\left(x_{2}\right), \cdots\right.$, $\left.\varphi\left(x_{n}\right)\right)=W_{2}\left(\varphi\left(x_{1}\right), \varphi\left(x_{2}\right), \cdots, \varphi\left(x_{n}\right)\right)$ holds in $G$.

For example, a regular semigroup in which the set of idempotents satisfies commutativity is an inverse semigroup firstly introduced by Vagner [9] under the term "generalized group" (see also [3], p. 25), and the structure of inverse semigroups was clarified by Preston [6] and [7]. A band (i.e., an idempotent semigroup) satisfying the identity $(C),(L . S),(R . S),(R),(L . N),(R . N)$ or $(N)$ is called a semilattice, left singular band, right singular band, rectangular band, left normal band, right normal band or normal band respectively, and the structure of these bands is completely determined by Kimura [4], McLean [5], Kimura and the author [17] and the author [12].

Now, we define an inversive semigroup as follows: A semigroup $G$ is called inversive if it satisfies the condition (1.2) and the following:

The set $I$ of all idempotents of $G$ is a subsemigroup of $G$. 
Of course, it is obvious that the set of idempotents of an inversive semigroup is a band. A semigroup satisfying the condition (1.2) is not necessarily a semigroup satisfying the condition (1.6). For example, a completely simple semigroup (for definition, see [3], p. 76) is a union of groups and hence is a semigroup satisfying the condition (1.2), but not necessarily a semigroup satisfying the condition (1.6). However, it should be noted that for commutative semigroups the condition (1.1) implies both the conditions (1.2) and (1.6) (hence, of course, the condition (1.2) implies the condition (1.6)). In other words, a commutative regular semigroup is inversive. Clifford [1] and the author [11] completely determined the structure of commutative regular semigroups and gave an explicit description of a method of constructing all possible commutative regular semigroups. It should be also noted that a noncommutative band (for example, a left singular band) is inversive but not an inverse semigroup. Now, each of an inverse semigroup, a commutative regular semigroup and a [left, right] normal band is of course a regular semigroup in which the set of idempotents satisfies a permutation identity. By a generalized inverse semigroup, hereafter we shall mean a regular semigroup in which the set of idempotents satisfies a permutation identity. Special kinds of generalized inverse semigroups have been studied by many papers, but no general structure theorem for generalized inverse semigroups has been established so far as we know. In the following sections we shall study generalized inverse semigroups, and establish a structure theorem for these semigroups and also present some relevant matters. Any notation and terminology should be referred to [3], unless otherwise stated.

2. Generalized inverse semigroups. Let $S$ be a regular semigroup. Then for each element $a$ of $S$, there exists an element $a^{*}$ such that $a a^{*} a=a$ and $a^{*} a a^{*}=a^{*}$ (see [3], p. 27). Such an element $a^{*}$ is called an inverse of $a$. For a given element $a$ of $S$, an inverse of $a$ is not necessarily unique. An inverse of $a$ is unique for every element $a$ of $S$ if and only if $S$ is an inverse semigroup (see [3], p. 28). In this case, we shall denote the inverse of $a$ by $a^{-1}$. At first we shall show several lemmas.

LEMMA 1. Let $S$ be a regular semigroup in which the set $B$ of $i d e m p o t e n t s$ is a normal band. Then for any elements $a, b$ of $S$ and for any elements $e, f$ of $B$, aefb $=a f e b$.

Proof. Let $a^{*}, b^{*}$ be inverses of $a, b$ respectively. Then, $a^{*} a$ and $b b^{*}$ are idempotents. Hence,

$$
a e f b=a\left(\left(a^{*} a\right) e f\left(b b^{*}\right)\right) b=a\left(\left(a^{*} a\right) f e\left(b b^{*}\right)\right) b=\left(a a^{*} a\right) f e\left(b b^{*} b\right)=a f e b .
$$


Lemma 2. (1) If a regular semigroup $S$ satisfies the following condition (2.1), then the set of idempotents of $S$ is a band:

(2.1) For any elements $a, b$ of $S$ and for any inverses $a^{*}$ of $a$ and $b^{*}$ of $b$, the element $b^{*} a^{*}$ is an inverse of $a b$.

(2) If the set of idempotents of a regular semigroup $S$ is a normal band, then $S$ satisfies the condition (2.1).

Proof. (1) Let $e, f$ be idempotents of $S$. Since $e, f$ are inverses of $e, f$ themselves respectively, by the assumption the element $f e$ is an inverse of ef. Hence, efef $=e f f e e f=e f$. That is, ef is an idempotent. Therefore, the set of idempotents of $S$ is a subsemigroup of $S$.

(2) Let $a, b$ be elements of $S$, and $a^{*}, b^{*}$ inverses of $a, b$ respectively. Then, $a a^{*}, a^{*} a, b b^{*}, b^{*} b$ are idempotents of $S$. Since the set $B$ of idempotents of $S$ is a normal band, it follows from Lemma 1 that $a b b^{*} a^{*} a b=a a^{*} a b b^{*} b=a b$ and $b^{*} a^{*} a b b^{*} a^{*}=b^{*} b b^{*} a^{*} a a^{*}=b^{*} a^{*}$. Hence $b^{*} a^{*}$ is an inverse of $a b$.

Lemma 3. If the set $B$ of idempotents of a regular semigroup $S$ satisfies a permutation identity, then $B$ is a normal band.

Proof. Let $S$ be a regular semigroup in which the set $B$ of idempotents satisfies a permutation identity

$$
x_{1} x_{2} \cdots x_{n}=x_{p_{1}} x_{p_{2}} \cdots x_{p_{n}} .
$$

Since $\left(p_{1}, p_{2}, \cdots, p_{n}\right)$ is a nontrivial permutation of $(1,2, \cdots, n)$, there exists $j$ such that $p_{j} \neq j$ and $p_{i}=i$ for all $i<j$. Let $p_{j}=s$ and $j=p_{k}$. Then, clearly $s>j$ ( $j, s$ might be $1, n$ respectively). Therefore, (2.2) has a form

$$
x_{1} x_{2} \cdots x_{j-1} x_{j} \cdots x_{s} \cdots x_{n}=x_{p_{1}} x_{p_{2}} \cdots x_{p_{j-1}} x_{p_{j}} \cdots x_{p_{k}} \cdots x_{p_{n}} .
$$

At first, we prove that $B$ is a band. Let $e, f$ be elements of $B$. Consider the mapping $\varphi:\left\{x_{1}, x_{2}, \cdots, x_{n}\right\} \rightarrow B$ such that $\varphi\left(x_{t}\right)=e$ for $1 \leqq t \leqq j$ and $\varphi\left(x_{t}\right)=f$ for $j+1 \leqq t \leqq n$. Then, $\varphi\left(x_{1}\right) \varphi\left(x_{2}\right) \cdots \varphi\left(x_{n}\right)=$ $\varphi\left(x_{p_{1}}\right) \varphi\left(x_{p_{2}}\right) \cdots \varphi\left(x_{p_{n}}\right)$ becomes ef =efef, ef $=f e f$, ef $=$ efe or $e f=f e$. If $e f=e f e$ or $e f=f e f$, then $e f=e f e f$. Therefore, in all cases $e f$ is an idempotent. Hence, $B$ is a band. Since $B$ satisfies the permutation identity (2.2), $B$ is normal (see [12] or [17]).

The following is a special case of a theorem given by Clifford [1] (see also McLean [5]):

For any band $B$, there exist a semilattice $\Gamma$ and a collection of 
rectangular bands, $\left\{B_{\gamma}: \gamma \in \Gamma\right\}$, such that

(1) $B=\cup\left\{B_{\gamma}: r \in \Gamma\right\}$,

(2) $B_{\alpha} \cap B_{\beta}=\square$ for $\alpha \neq \beta$ and

(3) $B_{\alpha} B_{\beta} \subset B_{\alpha \beta}$ for all $\alpha, \beta \in \Gamma$.

Further such a decomposition of $B$ is unique. Accordingly $\Gamma$ is unique up to isomorphism, and so are the $B_{\gamma}$ 's.

The $\Gamma$ above is called the structure semilattice of $B$, and $B_{\gamma}$ is called the $\gamma$-kernel of $B$. Further, this decomposition is called the structure decomposition of $B$, and denoted by $B \sim \sum\left\{B_{\gamma}: \gamma \in \Gamma\right\}$.

Now it can be proved that a band $B$ is normal if and only if it satisfies the identity $x y z x=x z y x$ or $x y x z x=x z x y x$. The "only if" part is obvious. Assume that $B$ satisfies the identity $x y x z x=x z x y x$. Let $B \sim \sum\left\{B_{\gamma}: \gamma \in \Gamma\right\}$ be the structure decomposition of $B$. Take elements $e, f, h$ from $B$, and suppose that $e \in B_{\alpha}, f \in B_{\beta}$ and $h \in B_{\gamma}$. Since $B$ satisfies the identity $x y x z x=x z x y x$, we have $e(f h f) e(h f h) e=$ $e(h f h) e(f h f) e$. Since efhfe,ehf,fhe $\in B_{\alpha \beta \gamma}$ and since $B_{\alpha \beta \gamma}$ is rectangular,

$$
e(f h f) e(h f h) e=e f h f e(e h f) f h e=e(f h f e f) h e=e(f e f h f) h e=e f h e .
$$

Similarly, we have $e(h f h) e(f h f) e=e h f e$. Hence, efhe = ehfe for any elements $e, f, h$ of $B$. Thus, $B$ satisfies the identity $x y z x=x z y x$. Take any elements $a, b, c, d$ from $B$, and suppose that $a \in B_{\alpha}, b \in B_{\beta}$, $c \in B_{\gamma}, d \in B_{\delta}$. Then, $a b c d=a b c a b c d=a c b a b c d=a c b b a c d=a c b a c d=$ $a c b a c d d b a c d=a c b d a c d b a c d=a c b d a c d b b a c d=a c b d a c d b a c b d$. Since $a c b d$ and $a c d b$ are contained in the same kernel $B_{\alpha \beta \gamma \delta}$ and since $B_{\alpha \beta \gamma \delta}$ is rectangular, $a c b d a c d b a c b d=a c b d a c b d=a c b d$. Hence, $a b c d=a c b d$. This means that $B$ is normal.

LEMMA 4. Let $S$ be a regular semigroup in which the set $B$ of idempotents is a band.

(1) If $B$ is a normal band, then the intersection $a S \cap S b(=a S b)$ of a principal right ideal aS and a principal left ideal $S b$ is a subsemigroup in which any two of the idempotents commute. In particular, $e S e$ is an inverse semigroup for any idempotent e of $S$.

(2) If every efe, where e, $f$ are elements of $B$, has precisely one inverse in eSe, then $B$ is a normal band.

Proof. (1) Let $x \in a S \cap S b$. Then, there exist $x_{1}, x_{2}$ such that $x=a x_{1}$ and $x=x_{2} b$. Let $a^{*}, b^{*}$ be inverses of $a, b$ respectively. Then, $a a^{*} x=a a^{*} a x_{1}=a x_{1}=x$ and $x b^{*} b=x_{2} b b^{*} b=x_{2} b=x$. Hence, $x=$ $a a^{*} x b^{*} b \in a S b$. Since $a S b \subset a S \cap S b$ is obvious, we obtain $a S \cap S b=$ $a S b$. Let $a a^{*}=e$ and $b^{*} b=f$. Then, $e$ and $f$ are idempotents and $a S b=e S f$. Let egf and ehf be any two idempotents of eSf. Then 
$e g f e h f=e(e g f)(e h f) f=e(e h f)(e g f) f=e h f e g f$. Hence, any two idempotents of $a S b$ commute. Next, we prove that $e S e$ is an inverse semigroup for an idempotent $e$ of $S$. Let exe be an element of $e S e$, and $x^{*}$ an inverse of $x$ in $S$. Then, $x^{*} e x x^{*} e x=x^{*} x x^{*} e e x=x^{*} e x$ since $x x^{*}$ is an idempotent and since $B$ is normal. Hence, $x^{*} e x$ is an idempotent. Now, exeex*eexe $=e\left(x e x^{*}\right) e x e=e e\left(x e x^{*}\right) x e=e x e\left(x^{*} x\right) e=e x x^{*} x e=e x e$. Hence $e S e$ is a regular semigroup. Since $e S e$ is a regular semigroup in which any two idempotents commute, it is an inverse semigroup (see [3], p. 28).

(2) Let $B \sim \sum\left\{B_{\gamma}: \gamma \in \Gamma\right\}$ be the structure decomposition of $B$. Take elements $e, f, h$ from $B$, and suppose that $e \in B_{\alpha}, f \in B_{\beta}$ and $h \in B_{\gamma}$. Then both the elements efhe and ehfe are contained in $B_{\alpha \beta \gamma}$. Since $B_{\alpha \beta \gamma}$ is rectangular, efheehfeefhe $=$ efhe and ehfeefheehfe = ehfe, that is, ehfe is an inverse of efhe. Since $e f h e$ is an idempotent, efhe itself is an inverse of efhe. Hence efhe $=e h f e$ follows from our assumption that ef he has precisely one inverse in $e S e$.

THEOREM 1. The following five conditions on a regular semigroup $S$ are equivalent:

(1) $S$ is a generalized inverse semigroup;

(2) The set of idempotents of $S$ is a normal band;

(3) The set of idempotents of $S$ is a band, and the intersection $a S \cap S b(=a S b)$ of a principal right ideal aS and a principal left ideal $\mathrm{Sb}$ is a subsemigroup in which any two of the idempotents commute;

(4) The set of idempotents of $S$ is a band, and $e S e$ is an inverse subsemigroup for any idempotent $e$ of $S$;

(5) $S$ satisfies the condition (2.1). Further every efe, where $e, f$ are idempotents of $S$, has precisely one inverse in eSe.

Proof. By Lemma 3, clearly (1) is equivalent to (2). Further, by Lemma 4, (2) implies (3) and (4). Conversely, suppose that $S$ satisfies (3). Let $B$ be the set of idempotents of $S$, and $e, f, h$ any elements of $B$. Then since $e f e$ and ehe are elements of $e S e$ and since any two of the idempotents of $e S e$ commute, we have efeehe = eheefe, that is, efehe =ehefe. Hence $B$ is normal. Similarly, we can prove that (4) implies (2). Next, suppose that $S$ satisfies (2). By (2) of Lemma 2, $S$ satisfies the condition (2.1). Since $S$ satisfies (2), it satisfies also (4). Hence, eSe is an inverse semigroup for any idempotent $e$ of $S$. Let $f$ be any idempotent of $S$. Since $e S e$ is an inverse semigroup and since efe is an element of $e S e$, the element efe has precisely one inverse in $e S e$. Thus, (2) implies (5). Conversely, let $S$ satisfy (5). It follows from (1) of Lemma 2 that the set $B$ of idempotents of $S$ is a band. Hence by (2) of Lemma $4, B$ is a normal 
band. Therefore, (5) implies (2).

Next, we shall present a structure theorem for generalized inverse semigroups. At first, we introduce the concept of a quasi-direct product: Let $\Omega$ be an inverse semigroup, and $\Gamma$ the set of idempotents of $\Omega$. Then $\Gamma$ is a commutative idempotent subsemigroup, i.e., a semilattice contained in $\Omega$. Hereafter, we shall call $\Gamma$ the basic semilattice of $\Omega$. Let $L$ and $R$ be a left normal band and a right normal band, having structure decompositions $L \sim \sum\left\{L_{\gamma}: \gamma \in \Gamma\right\}$ and $R \sim \sum\left\{R_{\gamma}: \gamma \in \Gamma\right\}$ respectively. In this case, each $L_{\gamma}$ is a left singular band and each $R_{\gamma}$ is a right singular band (see [12] and [17]). Let $S=\left\{(e, \xi, f): \xi \in \Omega, e \in L_{\xi \xi-1}, f \in R_{\xi-1_{\xi}}\right\}$, and define multiplication $\circ$ in $S$ as follows:

$$
(e, \xi, f) \circ(g, \eta, h)=(e u, \xi \eta, v h),
$$

where $u \in L_{\xi \eta(\xi \eta)-1}$ and $v \in R_{(\xi \eta)^{-1} \xi \eta}$. Such multiplication $\circ$ is well-defined. In fact:

$$
e u \in e L_{\xi \eta(\xi \eta)^{-1}} \subset L_{\xi \xi^{-1}} L_{\xi \eta(\xi \eta)^{-1}} \subset L_{\xi^{-1} \xi^{-1} \eta^{-1} \xi^{-1}}=L_{\xi \eta \eta^{-1} \xi^{-1}}=L_{\xi \eta(\xi \eta)^{-1}}
$$

and

$$
v h \in R_{(\xi \eta)^{-1} \xi \eta} h \subset R_{\eta^{-1} \xi^{-1 \xi \eta}} R_{\eta^{-1} \eta} \subset R_{\eta^{-1} \xi^{-1 \xi \eta \eta^{-1} \eta}}=R_{\eta^{-1} \xi^{-1} \xi \eta}=R_{(\xi \eta)^{-1 \xi \eta}} .
$$

Hence, $(e u, \xi \eta, v h) \in S$. Let $u_{1} \in L_{\xi \eta(\xi \eta)-1}$ and $v_{1} \in R_{(\xi \eta)-1 \xi \eta \eta}$. Since $e u$ and $e u_{1}$ are contained in $L_{\xi \eta(\xi \eta)^{-1}}, L_{\xi \eta(\xi \eta)^{-1}}$ is left singular and $L$ is left normal, we have $e u=e u e u_{1}=e e u_{1} u=e u_{1} u=e u_{1}$. Similarly, we have $v_{1} h=v h$. Hence $(e u, \xi \eta, v h)=\left(e u_{1}, \xi \eta, v_{1} h\right)$, that is, $(e, \xi, f) \circ(g, \eta, h)$ is uniquely determined by $(e, \xi, f)$ and $(g, \eta, h)$.

Now by simple calculation we can easily prove the following lemma:

Lemma 5. The resulting system $S(\circ)$ is a regular semigroup in which the set of idempotents is a normal band. Hence, $S(\circ)$ is a generalized inverse semigroup.

Proof. At first, we shall show that $S(\circ)$ satisfies the associative law and hence is a semigroup. Let $(e, \xi, f),(g, \eta, h)$ and $(i, \rho, j)$ be elements of $S(\circ)$. Then,

$$
\{(e, \xi, f) \circ(g, \eta, h)\} \circ(i, \rho, j)=(e u, \xi \eta, v h) \circ(i, \rho, j)=(e u w, \xi \eta \rho, x j),
$$

where $u \in L_{\xi \eta(\xi \eta)-1}, v \in R_{(\xi \eta)-1 \xi \eta}, w \in L_{(\xi \eta \rho)(\xi \eta \rho)-1}$ and $x \in R_{(\xi \eta \rho)-1 \xi \eta \rho}$. Since $L$ is left normal, euw $=e(u w) w=e w(u w)$. Further,

$$
e w \in L_{\xi \xi^{-1}} L_{(\xi \eta \rho)(\xi \eta \rho)-1}=L_{\xi \xi^{-1}{ }^{-1} \eta \rho(\xi \eta \rho)-1}=L_{\xi \eta \rho(\xi \eta \rho)-1}
$$

and 
$u w \in L_{\xi \eta(\xi \eta)^{-1}} L_{(\xi \eta \rho)(\xi \eta \rho)-1}=L_{\xi \eta \eta^{-1} \xi-1 \xi \eta \rho(\xi \eta \rho)-1}=L_{\xi \xi-1 \xi \eta \eta \eta^{-1} \eta \rho(\xi \eta \rho)-1}=L_{\xi \eta \rho(\xi \eta \rho)-1}$.

Since $L_{\xi \eta \rho(\xi \eta \rho)-1}$ is left singular, euw $=(e w)(u w)=e w$. Hence,

$$
\{(e, \xi, f) \circ(g, \eta, h)\} \circ(i, \rho, j)=(e w, \xi \eta \rho, x j) .
$$

On the other hand,

$$
(e, \xi, f) \circ\{(g, \eta, h) \circ(i, \rho, j)\}=(e, \xi, f) \circ(g k, \eta \rho, s j)=(e w, \xi \eta \rho, x s j),
$$

where $k \in L_{\eta \rho(\eta \rho)-1}$ and $s \in R_{(\eta \rho)-1}$. By the same method used above, we can easily prove that $x s j=x j$. Hence,

$(e, \xi, f) \circ\{(g, \eta, h) \circ(i, \rho, j)\}=(e w, \xi \eta \rho, x j)=\{(e, \xi, f) \circ(g, \eta, h)\} \circ(i, \rho, j)$.

Thus $S(\circ)$ is a semigroup. Take $(e, \xi, f)$ and $\left(g, \xi^{-1}, h\right)$ from $S(\circ)$. Then,

$$
(e, \xi, f) \circ\left(g, \xi^{-1}, h\right) \circ(e, \xi, f)=\left(e t, \xi \xi^{-1} \xi, n f\right),
$$

where $t \in L_{\xi \xi^{-1} \xi_{\left(\xi \xi^{-1} \xi^{-1}\right.}}=L_{\xi \xi^{-1}}$ and $n \in R_{\left.\left(\xi \xi^{-1}\right)^{-1}\right)_{\xi \xi^{-1}}}=R_{\xi^{-1} \xi}$. Since $e, t \epsilon$ $L_{\xi^{-1}}, n, f \in R_{\xi^{-1} \xi}$ and since $L_{\xi^{-1}}, R_{\xi^{-1} \xi}$ are left singular and right singular respectively, it follows that $e t=e$ and $n f=f$. Therefore,

$$
(e, \xi, f) \circ\left(g, \xi^{-1}, h\right) \circ(e, \xi, f)=(e, \xi, f) .
$$

This means that $S(\circ)$ is a regular semigroup. Next, we prove that the set $B$ of idempotents of $S(\circ)$ is a normal band. If $(e, \xi, f)$ is an element of $B$, then $(e, \xi, f)=(e, \xi, f) \circ(e, \xi, f)=\left(e u, \xi^{2}, v f\right)$, where $u \in L_{\xi^{2}\left(\xi^{2}\right)-1}$ and $v \in R_{\left(\xi^{2}\right)-1 \xi^{2}}$. Hence $\xi=\xi^{2}$. Conversely, let $\xi$ be an idempotent of $\Omega$ and $e, f$ elements of $L_{\xi^{-1}}\left(=L_{\xi}\right)$ and $R_{\xi^{-1} \xi^{-}}\left(=R_{\xi}\right)$ respectively. Then $(e, \xi, f) \circ(e, \xi, f)=(e u, \xi, v f)$, where $u \in L_{\xi^{-1}}\left(=L_{\xi}\right)$ and $v \in R_{\xi^{-1} 1_{\xi}}\left(=R_{\xi}\right)$. Since $e, u \in L_{\xi}$ and $v, f \in R_{\xi}$, we have $e u=e$ and $v f=f$. Therefore $(e, \xi, f) \circ(e, \xi, f)=(e, \xi, f)$, that is, $(e, \xi, f)$ is an idempotent of $S(\circ)$. Hence, $B=\left\{(e, \xi, f): \xi \in \Gamma, e \in L_{\xi}, f \in R_{\xi}\right\}$. It is obvious that $B$ is a band. Take three elements $\left(e_{1}, \xi_{1}, f_{1}\right),\left(e_{2}, \xi_{2}, f_{2}\right)$ and $\left(e_{3}, \xi_{3}, f_{3}\right)$ from $B$. Since $\Gamma$ is a semilattice, we have

$$
\begin{aligned}
& \left(e_{1}, \xi_{1}, f_{1}\right) \circ\left(e_{2}, \xi_{2}, f_{2}\right) \circ\left(e_{3}, \xi_{3}, f_{3}\right) \circ\left(e_{1}, \xi_{1}, f_{1}\right)=\left(e_{1} u, \xi_{1} \xi_{2} \xi_{3} \xi_{1}, v f_{1}\right) \\
& \quad=\left(e_{1} u, \xi_{1} \xi_{3} \xi_{2} \xi_{1}, v f_{1}\right)=\left(e_{1}, \xi_{1}, f_{1}\right) \circ\left(e_{3}, \xi_{3}, f_{3}\right) \circ\left(e_{2}, \xi_{2}, f_{2}\right) \circ\left(e_{1}, \xi_{1}, f_{1}\right),
\end{aligned}
$$

where $u \in L_{\hat{\xi}_{1} \hat{\xi}_{2} \hat{\xi}_{3} \xi_{1}}\left(=L_{\hat{\xi}_{1} \hat{\xi}_{3} \xi_{2} \xi_{1}}\right)$ and $v \in R_{\xi_{1} \hat{\xi}_{2} \hat{\xi}_{3} \hat{\xi}_{1}}\left(=R_{\xi_{1} \hat{\xi}_{3} \xi_{2} \xi_{1}}\right)$. This means that $B$ is normal.

We shall call $S(\circ)$ in Lemma 5 the quasi-direct product of $L, \Omega$ and $R$ with respect to $\Gamma$, and denote it by $Q(L \otimes \Omega \otimes R ; \Gamma)$. Now, let $S$ be a generalized inverse semigroup and let $B$ be the normal band consisting of all idempotents of $S$. Let $B \sim \sum\left\{B_{\gamma}: \gamma \in \Gamma\right\}$ be the structure decomposition of $B$. 
Let us define a relation $\mathfrak{D}$ on $S$ as follows:

(2.3) $x \mathfrak{D} y$ if and only if $\left\{x^{*} ; x^{*} \in S\right.$ and $x^{*}$ is an inverse of $\left.x\right\}$ $=\left\{y^{*}: y^{*} \in S\right.$ and $y^{*}$ is an inverse of $\left.y\right\}$.

Then $\mathfrak{D}$ is a congruence on $S$. In fact: $\mathfrak{D}$ is clearly an equivalence relation on $S$. Suppose that $x \mathscr{D} y$ and $c$ is an element of $S$. Suppose also that $t$ is an inverse of $c x$. Let $x^{*}$ be an inverse of $x$. Since $x \mathfrak{D} y, x^{*}$ is also an inverse of $y$. Therefore, $y x^{*} y=y, x^{*} y x^{*}=x^{*}$ and each of the elements $y x^{*}, x^{*} y, x x^{*}$ and $x^{*} x$ is an idempotent. Now,

$$
\begin{aligned}
& c x t c x=c x x^{*} x t c x x^{*} x=c x x^{*} y x^{*} x t c x x^{*} y x^{*} x=c y x^{*} x x^{*} x t c y x^{*} x x^{*} x \\
& =c y x^{*} x t c y x^{*} x=c y x^{*} y x^{*} x t c y x^{*} x=c y x^{*} x x^{*} y t c y x^{*} x=c y t c y x^{*} x .
\end{aligned}
$$

Since $c x t c x=c x, \operatorname{cyt} c y x^{*} x=c x$. Hence,

$$
\text { cytcy }=\text { cytcy } x^{*} x x^{*} y=c x x^{*} y=c x x^{*} y x^{*} y=c y x^{*} x x^{*} y=c y .
$$

Further,

$$
\begin{aligned}
t c y t & =t c y x^{*} y t=t c y x^{*} x x^{*} y t=t c x x^{*} y x^{*} y t=t c x x^{*} y t \\
& =t c x x^{*} x x^{*} y t=t c x x^{*} y x^{*} x t=t c x t=t .
\end{aligned}
$$

Hence, $t$ is an inverse of $c y$. Similarly, any inverse of $c y$ is also an inverse of $c x$. This means that cxDcy. By the same method, we can prove that $x \mathfrak{D} y$ implies $x c \mathfrak{D} y c$ for any element $c$ of $S$. That is, D is a congruence on $S$.

Next, consider the restriction $\mathfrak{D}_{B}$ of $\mathfrak{D}$ to $B$. Let $e, f$ be elements of $B$. It is clear that $e \mathfrak{D}_{B} f$ implies $e f e=e$ and $f e f=f$. Conversely, suppose that $e f e=e$ and $f e f=f$. For any inverse $f^{*}$ of $f, e f^{*} e=$ efef*efe $=e e f f^{*} f e e$ (by Lemma 1) $=e f e=e$ and $f^{*} e f^{*}=f^{*} e f e f^{*}=$ $f^{*}$ efffef $f^{*}=f^{*}$ fefeff $f^{*}$ (by Lemma 1$)=f^{*} f f f^{*}=f^{*} f f^{*}=f^{*}$. Hence, $f^{*}$ is also an inverse of $e$. Similarly, any inverse of $e$ is also an inverse of $f$. Hence $e \mathfrak{D}_{B} f$. Thus for any $e, f \in B, e \mathfrak{D}_{B} f$ if and only if $e f e=e$ and $f e f=f$. This means that $\mathfrak{D}_{B}$ gives the structure decomposition of $B$ and that the factor semigroup $B / \mathscr{D}_{B}$ of $B \bmod \mathfrak{D}_{B}$ is $\left\{B_{\gamma}: \gamma \in \Gamma\right\}$ (hence of course, $B / \mathscr{D}_{B}$ is a semilattice such that, for any $\alpha, \beta \in \Gamma, B_{\alpha} \cdot B_{\beta}=B_{\alpha \beta}$, where - is multiplication in $B / \mathscr{D}_{B}$; see [1], [5] and [12]). We also define relations $\mathfrak{R}, \mathfrak{\&}$ on $B$ as follows:

$$
\begin{aligned}
& e \Re f \text { if and only if } e f=f \text { and } f e=e . \\
& e \Re f \text { if and only if } e f=e \text { and } f e=f .
\end{aligned}
$$

Then $\mathfrak{R}$ and $\mathfrak{R}$ are clearly congruences on $B$ satisfying $\mathfrak{R}, \mathfrak{\Omega} \leqq \mathfrak{D}_{B}$, and the factor semigroups $B / \Re, B / \mathbb{R}$ are bands, having $B / \Re \sim \sum\left\{B_{\gamma} / R_{\gamma}: B_{\gamma} \in\right.$ $\left.B / \mathfrak{D}_{B}\right\}$ and $B / \mathbb{R} \sim \sum\left\{B_{\gamma} / \mathbb{R}_{\gamma}: B_{\gamma} \in B / \mathfrak{D}_{B}\right\}$ as their structure decompositions 
respectively, where $\Re_{\gamma}$ and $\mathbb{R}_{\gamma}$ are the restrictions of $\Re$ and $\mathbb{L}$ to the $\gamma$-kernel $B_{\gamma}$ of $B$.

By using these results, we obtain the following

LEMMA 6. Let $S$ be a generalized inverse semigroup, and $B$ the normal band consisting of all idempotents of $S$. Let $B \sim \sum\left\{B_{\gamma}: \gamma \in \Gamma\right\}$ be the structure decomposition of $B$. Let $\mathfrak{D}, \mathfrak{R}$ and $\&$ be the congruences defined by (2.3), (2.4) and (2.5) respectively. Let $\mathfrak{D}_{B}$ be the restriction of $\mathfrak{D}$ to $B$, and for any $\gamma$ of $\Gamma$ let $\Re_{\gamma}$ and $\mathfrak{S}_{\gamma}$ be the restrictions of $\mathfrak{R}$ and $\mathbb{Q}$ to the $\gamma$-kernel $B_{\gamma}$ of $B$ respectively. Then,

(1) $S / \mathfrak{D}$ is an inverse semigroup having $B / \mathfrak{D}_{B}\left(=\left\{B_{\gamma}: \gamma \in \Gamma\right\}\right)$ as its basic semilattice, and $B / \Re$ and $B / \mathbb{R}$ are a left normal band and a right normal band, having $B / \Re \sim \sum\left\{B_{\gamma} / \Re_{\gamma}: B_{\gamma} \in B / \mathfrak{D}_{B}\right\}$ and $B / \mathbb{R} \sim \sum\left\{B_{\gamma} / \mathbb{R}_{\gamma}: B_{\gamma} \in B / \mathfrak{D}_{B}\right\}$ as their structure decompositions; and

(2) $S$ is isomorphic to the quasi-direct product $Q(B / \Re \otimes S / \mathfrak{D} \otimes$ $\left.B / 2 ; B / \mathfrak{D}_{B}\right)$.

Proof. (1) Let $\bar{x}$ denote the congruence class containing $x$ $\bmod \mathfrak{D}$, and let $\widetilde{e}, \widetilde{e}$ denote the congruence classes containing $e \bmod$ $\Re, \&$ respectively. At first, we prove that $S / \mathscr{D}$ is an inverse semigroup and has $B / \mathscr{D}_{B}$ as its basic semilattice. Let $a$ be an element of $S$, and $a^{*}$ an inverse of $a$. Then, $\bar{a} \bar{a}^{*} \bar{a}=\overline{a a^{*} a}=\bar{a}$. Hence $S / \mathfrak{D}$ is a regular semigroup. Suppose that $\bar{x}$ is an idempotent of $S / \mathfrak{D}$. Then $\bar{x}^{2}=\bar{x}$, that is, $x^{2} D x$. Let $x^{*}$ be an inverse of $x$. Then $x^{*} x^{2} x^{*}=x^{*}$, and hence $x x^{*} x x x^{*} x=x x^{*} x$, that is, $x^{2}=x$. Therefore, $x$ is an idempotent of $S$. Conversely, $\bar{x}$ is an idempotent if $x$ is an idempotent. Hence, it follows that the set of idempotents of $S / \mathscr{D}$ is $B / \mathscr{D}_{B}=$ $\left\{B_{\gamma}: \gamma \in \Gamma\right\}$. Since $B / \mathfrak{D}_{B}$ is a semilattice, $S / \mathfrak{D}$ is an inverse semigroup and has $B / \mathscr{D}_{B}$ as its basic semilattice. Next, we prove that $B / \Re$ is a left normal band having $B / \Re \sim \sum\left\{B_{\gamma} / \Re_{\gamma}: B_{\gamma} \in B / \mathfrak{D}_{B}\right\}$ as its structure decomposition. As was shown above, $B / \Re$ is a band having $B / \Re \sim$ $\sum\left\{B_{\gamma} / \Re_{\gamma}: B_{\gamma} \in B / \mathfrak{D}_{B}\right\}$ as its structure decomposition. Let $\widetilde{e}, \widetilde{f}, \widetilde{h}$ be elements of $B / \Re . \quad$ efhehf $=$ efehf $=e h f$ and $e h f e f h=e h e f h=e f h$.

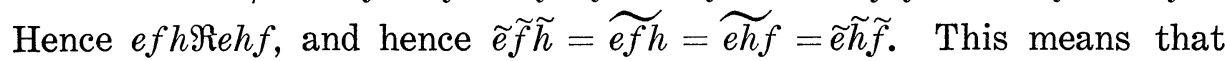
$B / \mathfrak{R}$ is left normal. Similarly, we can prove that $B / \mathcal{R}$ is a right normal band having $\left\{B_{\gamma} / \mathbb{R}_{\gamma}: B_{\gamma} \in B / \mathfrak{D}_{B}\right\}$ as its structure decomposition.

(2) Since the basic semilattice of $S / \mathscr{D}$ is $B / \mathscr{D}_{B}$ and since each of the structure semilattices of $B / \Re$ and $B / \Omega$ is $B / \mathscr{D}_{B}$, we can consider the quasi-direct product $Q\left(B / \Re \otimes S / \mathfrak{D} \otimes B / \Omega ; B / \mathfrak{D}_{B}\right)$. Now, define a mapping $\psi: S \rightarrow Q\left(B / \Re \otimes S / \mathfrak{D} \otimes B / \mathbb{R} ; B / \mathscr{D}_{B}\right)$ as follows:

$$
\psi(x)=\left(\widetilde{x x^{*}}, \bar{x}, \widetilde{x^{*} x}\right)
$$

where $x^{*}$ is an inverse of $x$. 
This mapping $\psi$ is well-defined. It can be proved as follows: Let $x^{*}$ be an inverse of $x$. Then $\bar{x}^{*}$ is the inverse of $\bar{x}$ in the inverse semigroup $S / \mathscr{D}$, and accordingly $\bar{x} \bar{x}^{*}, \bar{x}^{*} \bar{x}$ are elements of $B / \mathscr{D}_{B}$. Let $\bar{x} \bar{x}^{*}=B_{\xi}$ and $\bar{x}^{*} \bar{x}=B_{\eta}$. Since $\mathfrak{R} \leqq \mathfrak{D}_{B}$ and $\mathfrak{L} \leqq \mathfrak{D}_{B}$, it follows that $\widetilde{x x^{*}} \in B_{\xi} / \Re_{\xi}$ and $\widetilde{x^{*} x \in B_{\eta} / \Omega_{\eta}}$. Therefore, $\left(\widetilde{x x^{*}}, \bar{x}, \widetilde{x^{*} x}\right) \in Q(B / \Re \otimes S / \mathfrak{D} \otimes$ $\left.B / \mathbb{R} ; B / \mathscr{D}_{B}\right)$. Next, let $x_{1}^{*}, x_{2}^{*}$ be inverses of $x$. Then $\widetilde{x x_{1}^{*}}=\widetilde{x x_{2}^{*}}$ and $\widetilde{x_{1}^{*} x}=\underset{x_{2}^{*} x}{\widetilde{R}}$. Hence $\psi(x)$ is uniquely determined for every $x$ of $S$, that is, $\psi$ is a mapping of $S$ to $Q\left(B / \Re \otimes S / \mathscr{D} \otimes B / \mathcal{R} ; B / \mathscr{D}_{B}\right)$. Take any element $(\widetilde{e}, \bar{x}, \widetilde{\widetilde{f}})$ from $Q\left(B / \Re \otimes S / \mathscr{D} \otimes B / \mathfrak{R} ; B / \mathscr{D}_{B}\right)$. Let $x^{*}$ be an inverse

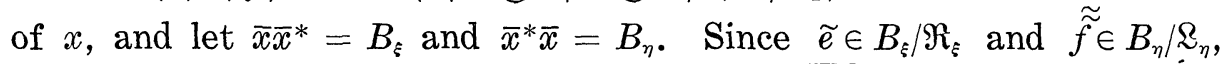
it follows that $e, x x^{*} \in B_{\xi}$ and $f, x^{*} x \in B_{\eta}$. $\overline{e x f}=\bar{e} \bar{x} \bar{f}=\overline{x x^{*}} * \bar{x} \overline{x^{*} x}=$ $\overline{x x^{*} x x^{*} x}=\bar{x}$. Since $f x^{*} e$ is an inverse of $e x f$, we have

$$
\widetilde{e x f f x^{*} e}=\widetilde{e x f x^{*} e}=\widetilde{e x x^{*} x f x^{*} x x^{*} e}=\widetilde{e x x^{*} x x^{*} x x^{*} e}=\widetilde{e x x^{*} e}=\widetilde{e} \text {. }
$$

Hence, $(\widetilde{e x f)(e x f})^{*}=\widetilde{e}$ for any inverse $(e x f)^{*}$ of exf. Similarly, we can prove that $\left(\widetilde{e x f)^{*}(e x f)}=\tilde{\widetilde{f}}\right.$ for any inverse $(e x f)^{*}$ of exf. Therefore,

$$
\psi(e x f)=\left(\widetilde{(e x f)(e x f)} * \overline{e x f}, \widetilde{(e x f)^{*}(e x f)}\right)=(\widetilde{e}, \bar{x}, \widetilde{\widetilde{f}}) .
$$

This means that $\psi$ is onto. Next, suppose that $\left.\widetilde{\left(x x^{*}\right.}, \bar{x}, \widetilde{x^{*} x}\right)=\left(\widetilde{y y^{*}}, \bar{y}\right.$, $\underset{y^{*} y}{\widetilde{\tau}}$. Since $\widetilde{x x^{*}}=\widetilde{y y^{*}}, \widetilde{x^{*} x}=\widetilde{y^{*} y}$ and $\bar{x}=\bar{y}$, we have $y y^{*} x x^{*}=x x^{*}$, $x^{*} x y^{*} y=x^{*} x$ and $y^{*} x y^{*}=y^{*}$. Hence,

$$
\begin{aligned}
x & =x x^{*} x=\left(y y^{*} x x^{*}\right) x=y y^{*} x\left(x^{*} x\right)=y y^{*} x\left(x^{*} x y^{*} y\right)=y y^{*}\left(x x^{*} x\right) y^{*} y \\
& =y\left(y^{*} x y^{*}\right) y=y y^{*} y=y .
\end{aligned}
$$

This means that $\psi$ is one-to-one. Finally, $\left.\psi(x y)=\widetilde{\left((x y)(x y)^{*}\right.}, \overline{x y}, \widetilde{(x y)^{*}(x y)}\right)$ where $(x y)^{*}$ is an inverse of $x y$. Since $y^{*} x^{*}$, where $y^{*}$ and $x^{*}$ are inverses of $y$ and $x$ respectively, is an inverse of $x y$, we have

$$
\begin{aligned}
& \left(\widetilde{(x y)(x y)^{*}}, \overline{x y}, \widetilde{(x y)^{*}(x y)}\right)=\left(\widetilde{x y y^{*} x^{*}}, \overline{x y}, \widetilde{y^{*} x^{*} x y}\right) \\
& =\left(\widetilde{x x^{*} x y y^{*} x^{*}}, \overline{x y}, \widetilde{y^{*} x^{*} x y y^{*} y}\right) \\
& =\left(\widetilde{x x^{*}}, \bar{x}, \widetilde{\widetilde{x^{*} x}}\right) \circ\left(\widetilde{y y^{*}}, \bar{y}, \underset{y^{*} y}{\widetilde{\tau}}\right)=\psi(x) \circ \psi(y) \text {. }
\end{aligned}
$$

Hence, $\psi$ is an isomorphism of $S$ onto $Q\left(B / \Re \otimes S / \mathfrak{D} \otimes B / \mathbb{R} ; B / \mathfrak{D}_{B}\right)$.

Summarizing Lemmas 5 and 6 , we obtain the following theorem:

THEOREM 2. A semigroup is a generalized inverse semigroup if and only if it is isomorphic to the quasi-direct product of a left normal band, an inverse semigroup and a right normal band. 
3. A structure theorem for $N$-inversive semigroups. As a special case of the $\S 2$, in this section we shall study the structure of regular semigroups satisfying permutation identities.

Let $S$ be a regular semigroup satisfying a permutation identity

$$
x_{1} x_{2} \cdots x_{n}=x_{p_{1}} x_{p_{2}} \cdots x_{p_{n}} \text {. }
$$

There exists $j$ such that $p_{j} \neq j$ and $p_{i}=i$ for all $i<j$. Let $p_{j}=s$ and $j=p_{k}$. Then, clearly $s>j$ ( $j, s$ might be $1, n$ respectively).

At first, we have:

LEMma 7. $S$ is an inversive semigroup in which the set of idempotents is a normal band.

Proof. It follows from Lemma 3 that the set of idempotents of $S$ is a normal band. Let $a^{*}$ be an inverse of an element $a$ of $S$. Put $a a^{*}=e$ and $a^{*} a=f$. Then, $e$ and $f$ are idempotents. We put $a^{*}$ and $a$ to the places $x_{s}$ and $x_{s-1}$ of (3.1) respectively, and $e$ to the other places $x_{i}$. Then, $e x_{1} x_{2} \cdots x_{j} \cdots x_{s} \cdots x_{n} e$ becomes $e a a^{*} e$ and $e x_{p_{1}} x_{p_{2}} \cdots x_{p_{j}} \cdots x_{p_{k}} \cdots x_{p_{n}} e$ becomes $e a^{*} a e$ or $e a^{*} e a e$. Since both $e a^{*} a e$ and $e a^{*} e a e$ are equal to $e f e$, we have $e=e f e$. Similarly, if we put $a$ and $a^{*}$ to the places $x_{s}$ and $x_{s-1}$ and $f$ to the other places $x_{i}$, then we have $f e f=f$. Let $e a^{*} f=x$. Then,

$$
\begin{aligned}
& a x=a\left(e a^{*} f\right)=(a f) e\left(f a^{*}\right) f=a(f e f) a^{*} f=a f a^{*} f=a a^{*} f=e f, \\
& x a=\left(e a^{*} f\right) a=e\left(a^{*} e\right) f(e a)=e a^{*}(e f e) a=e a^{*} e a=e a^{*} a=e f
\end{aligned}
$$

and $a x a=a\left(e a^{*} f\right) a=e f a=e f(e a)=(e f e) a=e a=a$. Hence, $S$ is inversive.

Let $G$ be an inversive semigroup. For any element $x$ of $G$, there exists an element $z$ such that $x z=z x$ and $x z x=x$. Further, we can prove that there exists one and only one element $y$ such that $x y=y x$, $x y x=x$ and $y x y=y$. In fact: Let $y=x z z$. Then, $x y=x(x z z)=$ $x z x z=x z, y x=(x z z) x=x z x z=x z, x y x=x z x=x$ and $y x y=(x z z) x z=$ $x z x z z=x z z=y$. Next, suppose that there exists another element $w$ such that $x w=w x, x w x=x$ and $w x w=w$. Then, $x y=(x w x) y=$ $w x y x=w(x y x)=w x=x w$, and hence $y=y x y=y x w=x y w=x w w=$ $w x w=w$. Therefore, such an element $y$ is unique. This $y$ is called the strict inverse of $x$, and is denoted by $x^{\prime}$.

For an inversive semigroup $M$ in which the set $N$ of idempotents is a normal band, we have the following lemmas:

LEMma 8. If $x x^{\prime}=e$ and if $f$ is an idempotent such that $f \leqq e$ (i.e., $f e=e f=f)$, then $f x=x f$. 
Proof. Let $f x(f x)^{\prime}=u$ and $x f(x f)^{\prime}=v$. Then $f u=u, u e=e u=u$, $v f \doteq v$ and $e v=v e=v$. Now, by using the normality of $N$, we have $f x=f x u=f x e f u e=f x e u f e=f x f$ and $x f=v x f=e v f e x f=e f v e x f=$ $f x f$. Hence $f x=x f$.

Lemma 9. If $a a^{\prime}=e$ and $b b^{\prime}=f$, then $(a b)^{\prime}=e b^{\prime} a^{\prime} f$ and $(a b)(a b)^{\prime}=$ $e f$.

Proof.

$$
\begin{aligned}
& a b\left(e b^{\prime} a^{\prime} f\right)=a b f e f b^{\prime} a^{\prime} f=a f e f b b^{\prime} a^{\prime} f=a e f e f e a^{\prime} f \\
&=e f e f e a a^{\prime} f=e f e f e f=e f ; \\
&\left(e b^{\prime} a^{\prime} f\right) a b=e b^{\prime} a^{\prime} e f e a b=e b^{\prime} a^{\prime} a e f e b=e b^{\prime} f e f e f b \\
&=e b^{\prime} b f e f e f=e f e f e f=e f ; \\
& a b\left(e b^{\prime} a^{\prime} f\right) a b=e f a b=e f e a b=a e f e b=a e f e f b=a e f b=a b ;
\end{aligned}
$$

and

$$
\left(e b^{\prime} a^{\prime} f\right) a b\left(e b^{\prime} a^{\prime} f\right)=e f\left(e b^{\prime} a^{\prime} f\right)=e f e f b^{\prime} a^{\prime} f=e f b^{\prime} a^{\prime} f=e b^{\prime} a^{\prime} f .
$$

Hence, $(a b)^{\prime}=e b^{\prime} a^{\prime} f$ and $(a b)(a b)^{\prime}=e f$.

LEMMA 10. Let $S$ be a regular semigroup satisfying a permutation identity. Then $x y=e y x f$ for any elements $x, y$ of $S$, where $x x^{\prime}=e$ and $y y^{\prime}=f$.

Proof. Let $S$ satisfy the above-mentioned identity (3.1). Putting $x$ and $y$ to the places $x_{j}$ and $x_{p_{j}}$ of (3.1) respectively and ef to the other places, ef $x_{1} x_{2} \cdots x_{j-1} x_{j} \cdots x_{s} \cdots x_{n}$ ef becomes efxyef or efxefyef, while $e$ ef $x_{p_{1}} x_{p_{2}} \cdots x_{p_{j-1}} x_{p_{j}} \cdots x_{p_{k}} \cdots x_{p_{n}}$ ef becomes efyxef or efyefxef. Since efxefyef $=$ efxyef $=x y$ follows from Lemma 8 and since

$$
\text { efyefxef }=\text { efyfefexef }=\text { efyxef }=\text { eyxf },
$$

we have $x y=e y x f$.

Now, we shall define some special inversive semigroups. Let $G$ be an inversive semigroup and $I$ the set of idempotents of $G$. Then $G$ is said to be $R$-inversive, $C$-inversive, $L . N$-inversive, $R$. $N$-inversive or $N$-inversive respectively, if it satisfies the corresponding identity $(R),(C),(L . N),(R . N)$ or $(N)$ given in $\S 1$. Moreover, $G$ is said to be weakly $R$-inversive, weakly $C$-inversive, weakly $L . N$-inversive, weakly $R . N$-inversive or weakly $N$-inversive respectively, if $I$ satisfies the corresponding identity $(R),(C),(L . N),(R . N)$ or $(N)$. 
REMARK. The structure of [weakly] $C$-inversive semigroups was completely determined by Clifford [1] and the author [11], while the structure of weakly $R$-inversive semigroups was determined by the author [10] (see also Thierrin [8]). In particular, the following is due to [10]: A semigroup is weakly $R$-inversive if and only if it is isomorphic to the direct product of a group and a rectangular band. Let $M$ be an $R$-inversive semigroup. Then $M \cong G \times T$, where $G$ is a group and $T$ is a rectangular band. Since $M$ satisfies rectangularity (accordingly $G \times T$ satisfies rectangularity),

$$
(g, t)=(1, t)(g, t)(1, t)=(1, t)
$$

for elements $(1, t),(g, t)$ of $G \times T$ where 1 is the identity element of $G$. Hence $(1, t)=(g, t)$, and hence $1=g$. This means that $G$ consists of a single element, that is, the element 1. Consequently, $M$ is a rectangular band. Conversely, any rectangular band is clearly an $R$-inversive semigroup. Therefore, we have the following result: $A$ semigroup is $R$-inversive if and only if it is a rectangular band.

It is obvious that any group satisfying a permutation identity is commutative. Further, any semigroup with an identity element is commutative if it satisfies a permutation identity. However, a regular semigroup satisfying a permutation identity is not necessarily commutative and is in general quite different from a commutative semigroup. This is easily seen from the fact that a rectangular band $R$ is an $N$-inversive semigroup (hence a regular semigroup satisfying a permutation identity), but any two elements of $R$ do not commute (see [3], p. 25). Now, there arises a question whether a regular semigroup satisfying a permutation identity is $N$-inversive. Next, we shall show that the answer to this question is in the affirmative, that is, that a regular semigroup satisfying a permutation identity is necessarily $N$-inversive. Accordingly, the concept of " $N$-inversive semigroup" coincides with the concept of "regular semigroup satisfying a permutation identity".

THEOREM 3. For a semigroup $S$, the following two conditions are equivalent:

(1) $S$ is regular and satisfies a permutation identity.

(2) $S$ is $N$-inversive.

Proof. Let $S$ be a regular semigroup satisfying a permutation identity. Let $x, y, z$ and $w$ be elements of $S$. By Lemma $7, S$ is weakly $N$-inversive. Let $x x^{\prime}=e, y y^{\prime}=f, z z^{\prime}=g$ and $w w^{\prime}=h$. Then, $z x y w=z e f x y e f w($ by Lemma 9$)=z e(f x y e) f w=z e y x f w($ by Lemma 10) $=$ $z e f y x e f w=z g e f f y x e e f h w=z g f e f y x e f e h w$ (by the normality of the 
idempotents of $S$ ) $=z$ feyxfew. By Lemma 9, $y x(y x)^{\prime}=f e$ and hence $y x f e=f e y x=y x$. Therefore, $z f e y x f e w=z y x w$. Hence $z x y w=z y x w$. This means that $S$ is $N$-inversive. It is obvious that the condition (2) implies the condition (1).

CoRollary. For regular semigroups, any permutation identity implies normality $x y z w=x z y w$.

REMARK. A semigroup satisfying a permutation identity is not necessarily a semigroup satisfying normality $x y z w=x z y w$. This can be seen from the following example: Let $a, b, c, d$ be four letters. Consider the set $\mathfrak{S}=\left\{\left(a_{1} a_{2} \cdots a_{r}\right): r \leqq 4, a_{i} \neq a_{j}\right.$ if $i \neq j, a_{k}=a, b, c$ or $d$ for all $1 \leqq k \leqq r\} \cup\{0\}$. Define multiplication $\circ$ in $\subseteq$ as follows:

$$
\left\{\begin{aligned}
&(1) \quad 0 \circ \alpha=\alpha \circ 0=0 \text { for all } \alpha \in \mathfrak{S}, \\
&(2) \quad\left(a_{1} a_{2} \cdots a_{r}\right) \circ\left(b_{1} b_{2} \cdots b_{s}\right)=\left(a_{1} a_{2} \cdots a_{r} b_{1} b_{2} \cdots b_{s}\right) \text { if } \\
&\left(a_{1} a_{2} \cdots a_{r}\right),\left(b_{1} b_{2} \cdots b_{s}\right) \in \mathfrak{S} \mid 0 \text { and } \\
& a_{1}, a_{2}, \cdots, a_{r}, b_{1}, b_{2}, \cdots, b_{s} \\
& \text { are all different, } \\
&= 0, \text { otherwise. }
\end{aligned}\right.
$$

Then, $\mathfrak{S}(\circ)$ is a semigroup which satisfies any permutation identity $x_{1} x_{2} \cdots x_{n}=x_{p_{1}} x_{p_{2}} \cdots x_{p_{n}}$ with $n>4$; since $\alpha_{1} \circ \alpha_{2} \circ \cdots \circ \alpha_{n}=0$ for any elements $\alpha_{1}, \alpha_{2}, \cdots, \alpha_{n} \in \mathfrak{S}$ if $n>4$. However $\mathfrak{S}(0)$ does not satisfy normality, since

$$
(a) \circ(b) \circ(c) \circ(d)=(a b c d) \neq(a c b d)=(a) \circ(c) \circ(b) \circ(d) .
$$

For inversive semigroups, we have the following:

THEOREM 4. An inversive semigroup $G$ is expressible as a semilattice of weakly $R$-inversive semigroups. That is, there exist a semilattice $\Gamma$ and a collection $\left\{G_{\gamma}: \gamma \in \Gamma\right\}$ of weakly $R$-inversive subsemigroups $G_{\gamma}$ such that

(1) $G=\cup\left\{G_{\gamma}: \gamma \in \Gamma\right\}$,

(2) $G_{\alpha} \cap G_{\beta}=\square$ for $\alpha \neq \beta$, and

(3) $G_{\alpha} G_{\beta} \subset G_{\alpha \beta}$ for all $\alpha, \beta \in \Gamma$.

Further $\Gamma$ is determined uniquely up to isomorphism, and accordingly so are the $G_{\gamma}$ 's.

Proof. From Clifford [1], an inversive semigroup $G$ is a semilattice $\Gamma$ of completely simple semigroups; that is, 


$$
\left\{\begin{array}{l}
(1) \quad G=\cup\left\{G_{\gamma}: \gamma \in \Gamma\right\}, \\
(2) \quad G_{\alpha} \cap G_{\beta}=\square \text { for } \alpha \neq \beta, \\
(3) \quad G_{\alpha} G_{\beta} \subset G_{\alpha \beta}, \alpha, \beta \in \Gamma,
\end{array}\right.
$$

where each $G_{\gamma}$ is a completely simple semigroup. Let $E_{\gamma}$ be the totality of idempotents of $G_{\gamma}$. Then, $E_{\gamma}$ is a subband of $G_{\gamma}$. Since a completely simple semigroup in which the set of idempotents is a band is isomorphic to the direct product of a group and a rectangular band and since a semigroup is isomorphic to the direct product of a group and a rectangular band if and only if it is weakly $R$-inversive, each $G_{\gamma}$ is a weakly $R$-inversive subsemigroup of $G$ (see [3] and [10[). Next suppose that there exists another decomposition of $G$ into a semilattice of weakly $R$-inversive semigroups, say

$$
\begin{cases}(1) & G=\cup\left\{G_{\xi}^{*}: \xi \in \Gamma^{*}\right\}, \\ (2) & G_{\zeta}^{*} \cap G_{\tau}^{*}=\square \text { for } \zeta \neq \tau, \\ (3) & G_{\zeta}^{*} G_{\tau}^{*} \subset G_{\zeta \tau}^{*}, \zeta, \tau \in \Gamma^{*},\end{cases}
$$

where each $G_{\xi}^{*}$ is a weakly $R$-inversive subsemigroup and $\Gamma^{*}$ is a semilattice.

Let $E_{\xi}^{*}$ be the totality of idempotents of $G_{\xi}^{*}$. Then, $E_{\xi}^{*}$ is a rectangular band contained in $G_{\xi}^{*}$. Let $I$ be the band of idempotents of $G$. Then,

$$
\left\{\begin{array}{l}
(1) \quad I=\cup\left\{E_{\gamma}: \gamma \in \Gamma\right\}, \\
(2) \quad E_{\alpha} \cap E_{\beta}=\square \text { for } \alpha \neq \beta, \\
(3) \quad E_{\alpha} E_{\beta} \subset E_{\alpha \beta},
\end{array}\right.
$$

and

$$
\left\{\begin{array}{l}
(1) \quad I=\cup\left\{E_{\xi}^{*}: \xi \in \Gamma^{*}\right\}, \\
(2) \quad E_{\zeta}^{*} \cap E_{\tau}^{*}=\square \text { for } \zeta \neq \tau, \\
(3) \quad E_{\zeta}^{*} E_{\tau}^{*} \subset E_{\zeta \tau}^{*}
\end{array}\right.
$$

are semilattice decompositions of $I$ into rectangular bands. According to McLean [3], such a decomposition of $I$ is unique. Hence, we can assume that $\Gamma=\Gamma^{*}$ and $E_{\gamma}=E_{\gamma}^{*}$ for all $\gamma \in \Gamma$. Now since two decompositions (I) and (II) are different, there exists $\alpha \in \Gamma$ such that $G_{\alpha} \neq G_{\alpha}^{*}$. Hence, there exists $\beta \in \Gamma(\alpha \neq \beta)$ such that $G_{\alpha}^{*} \cap G_{\beta} \neq \square$ or $G_{\alpha} \cap G_{\beta}^{*} \neq \square$. If $G_{\alpha}^{*} \cap G_{\beta} \ni x$, then $x^{\prime} \in G_{\alpha}^{*} \cap G_{\beta}$. Hence $x x^{\prime} \in G_{\alpha}^{*} \cap G_{\beta}$, and hence $x x^{\prime} \in E_{\alpha} \cap E_{\beta}$. Similarly, $G_{\alpha} \cap G_{\beta}^{*} \neq \square$ implies $E_{\alpha} \cap E_{\beta} \neq \square$. This is a contradiction. Hence, such a decomposition of $G$ is unique.

We shall call $\Gamma$ in Theorem 4 the structure semilattice of $G$, and $G_{\gamma}$ the $\gamma$-kernel of $G$. This is a generalization of the concepts of the structure semilattice and a kernel of a band defined in $\S 2$. 
Also, in this case we write $G \sim \sum\left\{G_{\gamma}: \gamma \in \Gamma\right\}$ and call it the structure decomposition of $G$.

According to [1], it follows that a [weakly] $C$-inversive semigroup $M$ is expressible as a semilattice $\Lambda$ of commutative groups [groups] $M_{2}$. (for a band of semigroups, see [2]). Therefore, in this case the structure semilattice of $M$ is $\Lambda$ and the $\lambda$-kernel of $M$ is the group $M_{\lambda}$. Further, the structure decomposition of $M$ is $M \sim \sum\left\{M_{\lambda}: \lambda \in \Lambda\right\}$. Let $G_{1}, G_{2}$ be inversive semigroups having $\Gamma$ as their structure semilattices, and let $G_{1} \sim \sum\left\{G_{1}^{\gamma}: \gamma \in \Gamma\right\}$ and $G_{2} \sim \sum\left\{G_{2}^{\gamma}: \gamma \in \Gamma\right\}$ be the structure decompositions of $G_{1}$ and $G_{2}$ respectively. Then the set $G=\cup\left\{G_{1}^{\gamma} \times G_{2}^{\gamma}: \gamma \in \Gamma\right\}$, where $G_{1}^{\gamma} \times G_{2}^{\gamma}$ is the direct product of $G_{1}^{\gamma}$ and $G_{2}^{\gamma}$, becomes a subdirect product of $G_{1}$ and $G_{2}$. Such a $G$ is called the spined product of $G_{1}$ and $G_{2}$ with respect to $\Gamma$, and denoted by $G_{1} \infty G_{2}(\Gamma)$.

Under these definitions, we have the following

Lemma 11. Let $S$ be an $N$-inversive semigroup having $\Gamma$ as its structure semilattice. Let $B$ be the normal band consisting of all idempotents of $S$. Then

(1) $B$ has $\Gamma$ as its structure semilattice, and

(2) there exists a C-inversive semigroup, having $\Gamma$ as its structure semilattice, such that $S$ is isomorphic to $C \infty B(\Gamma)$.

Proof. Let $S \sim \sum\left\{S_{\gamma}: \gamma \in \Gamma\right\}$ be the structure decomposition of $S$. Let $E_{\gamma}$ be the totality of all idempotents of $S_{\gamma}$. The structure decomposition of $B$ is clearly $B \sim \sum\left\{E_{\gamma}: \gamma \in \Gamma\right\}$. Now, we introduce a relation $R$ on $S$ as follows: $x R y$ if and only if $x, y \in S_{\gamma}$ for some $\gamma \in \Gamma$ and $x y^{\prime} \in E_{\gamma}$. Then, it is easy to see that $R$ is a congruence on $S$. Therefore, we can consider the factor semigroup $S / R$ of $S \bmod$ $R$. We denote the congruence class containing $x$ by $\bar{x}$, and put $\left\{\bar{x}_{\gamma}\right.$ : $\left.x_{\gamma} \in S_{\gamma}\right\}=G_{\gamma}$. Then, $S / R=\cup\left\{G_{\gamma}: \gamma \in \Gamma\right\}$ and $G_{\alpha} \cap G_{\beta}=\square$ for $\alpha \neq \beta$. It is easy to see that $G_{\gamma}$ is a group having $\bar{e}_{\gamma}, e_{\gamma} \in E_{\gamma}$, as its identity element. Let $\bar{x}_{\alpha}, \bar{x}_{\beta}$ be elements of $G_{\alpha}$ and $G_{\beta}$. Clearly, $\bar{x}_{\alpha} \bar{x}_{\beta}=\bar{x}_{\alpha} x_{\beta}$. Since $x_{\alpha} x_{\beta} \in S_{\alpha \beta}, \overline{x_{\alpha} x_{\beta}}$ is an element of $G_{\alpha \beta}$. Hence, $G_{\alpha} G_{\beta} \subset G_{\alpha \beta}$. Thus, the structure decomposition of $S / R$ is $S / R \sim \sum\left\{G_{\gamma}: \gamma \in \Gamma\right\}$. Next, we shall prove that $S / R$ is commutative. Let $\bar{x}_{\alpha}, \bar{y}_{\beta}$ be elements of $S / R$ where $\bar{x}_{\alpha} \in G_{\alpha}$ and $\bar{y}_{\beta} \in G_{\beta}$. Let $x_{\alpha} x_{\alpha}^{\prime}=e$ and $y_{\beta} y_{\beta}^{\prime}=f$. The elements $x_{\alpha} y_{\beta}$ and $y_{\beta} x_{\alpha}$ are contained in $S_{\alpha \beta}$, and $x_{\alpha} y_{\beta}\left(y_{\beta} x_{\alpha}\right)^{\prime}=x_{\alpha} y_{\beta} f x_{\alpha}^{\prime} y_{\beta}^{\prime} e=$ $x_{\alpha} y_{\beta} x_{\alpha}^{\prime} y_{\beta}^{\prime} e=x_{\alpha} x_{\alpha}^{\prime} y_{\beta} y_{\beta}^{\prime} e=e f e \in E_{\alpha \beta}$. Hence $x_{\alpha} y_{\beta} R y_{\beta} x_{\alpha}$, and hence $\bar{x}_{\alpha} \bar{y}_{\beta}=$ $\bar{y}_{\beta} \bar{x}_{\alpha}$. Thus, $S / R$ is commutative. Since $S / R$ is inversive and commutative, $S / R$ is $C$-inversive. Next, consider the spined product $S / R \propto B(\Gamma): S / R \infty B=\cup\left\{G_{\gamma} \times E_{\gamma}: \gamma \in \Gamma\right\}$. Define a mapping $\varphi$ of $S$ into $S / R \infty B(\Gamma)$ as follows: $\varphi(x)=\left(\bar{x}, x x^{\prime}\right), x \in S$. Then, $\varphi(x y)=(\overline{x y}$, $\left.x y(y x)^{\prime}\right)=\left(\bar{x} \bar{y}, x x^{\prime} y y^{\prime}\right)\left(\right.$ by Lemma 9) $=\left(\bar{x}, x x^{\prime}\right)\left(\bar{y}, y y^{\prime}\right)=\varphi(x) \varphi(y)$. Let 
$\left(\bar{x}, e_{\alpha}\right), e_{\alpha} \in E_{\alpha}$, be any element of $S / R \infty B(\Gamma)$. Then, $x \in S_{\alpha}$. Let $x x^{\prime}=f \in E_{\alpha}$. Since $\bar{e}_{\alpha}$ is the identity element of $G_{\alpha}$, we have $\overline{e_{\alpha} x e_{\alpha}}=$ $\bar{e}_{\alpha} \bar{x} \bar{e}_{\alpha}=\bar{x}$ and

$$
\begin{aligned}
e_{\alpha} x e_{\alpha}\left(e_{\alpha} x e_{\alpha}\right)^{\prime} & =e_{\alpha} x e_{\alpha} e_{\alpha}\left(x e_{\alpha}\right)^{\prime} e_{\alpha}^{\prime} f e_{x}=e_{\alpha} x e_{\alpha} f e_{\alpha}^{\prime} x^{\prime} e_{\alpha} f e_{\alpha} \\
& =e_{\alpha} x e_{\alpha} x^{\prime} e_{\alpha}=e_{\alpha} x x^{\prime} e_{\alpha}=e_{\alpha} .
\end{aligned}
$$

Hence, $\varphi\left(e_{\alpha} x e_{\alpha}\right)=\left(\overline{e_{\alpha} x e_{\alpha}}, e_{\alpha} x e_{\alpha}\left(e_{\alpha} x e_{\alpha}\right)^{\prime}\right)=\left(\bar{x}, e_{\alpha}\right)$. This means that $\varphi$ is an onto-mapping. Next, suppose that $\varphi(x)=\varphi(y)$. Then, $\left(\bar{x}, x x^{\prime}\right)=$ $\left(\bar{y}, y y^{\prime}\right)$. Hence, $x x^{\prime}=y y^{\prime}$ and there exists $S_{\alpha}$ such that $x, y \in S_{\alpha}$ and $x y^{\prime} \in E_{\alpha}$. Let $x x^{\prime}=y y^{\prime}=e$ and $x y^{\prime}=e_{\alpha}$. Then $\left(y x^{\prime}\right)^{\prime}=e x y^{\prime} e=e e_{\alpha} e=$ $e$. Hence $y x^{\prime}=e$. Similarly, $\left(x y^{\prime}\right)^{\prime}=e y x^{\prime} e=e e e=e$. Hence, $x y^{\prime}=e$. Therefore, $x x^{\prime}=y y^{\prime}$ implies $e x=x x^{\prime} x=x y^{\prime} y=e y$, and hence $x=y$. Thus, $\varphi$ is an isomorphism of $S$ onto $S / R \infty B(\Gamma)$.

Using Lemma 11, we obtain the following main theorem:

Theorem 5. (Structure theorem). A semigroup $S$ is isomorphic to the spined product of a $C$-inversive semigroup and a normal band if and only if $S$ is $N$-inversive.

Proof. The "if" part was proved in Lemma 11. We shall prove the "only if" part. Let $C$ be a $C$-inversive semigroup having structure decomposition $C \sim \sum\left\{C_{\gamma}: \gamma \in \Gamma\right\}$. Let $B$ be a normal band having structure decomposition $B \sim \sum\left\{E_{\gamma}: \gamma \in \Gamma\right\}$. Since the spined product of any two inversive semigroups is also inversive, the spined product $C \infty B(\Gamma)$ is inversive. Now, $C \infty B(\Gamma)=\bigcup\left\{C_{\gamma} \times E_{\gamma}: \gamma \in \Gamma\right\}$. Let $\left(a_{\gamma}, e_{\gamma}\right),\left(a_{\alpha}, e_{\alpha}\right),\left(a_{\beta}, e_{\beta}\right),\left(a_{\delta}, e_{\delta}\right)$ be four elements of $C \infty B(\Gamma)$. Then,

$$
\left(a_{\gamma}, e_{\gamma}\right)\left(a_{\alpha}, e_{\alpha}\right)\left(a_{\beta}, e_{\beta}\right)\left(a_{\delta}, e_{\delta}\right)=\left(a_{\gamma} a_{\alpha} a_{\beta} a_{\delta}, e_{\gamma} e_{\alpha} e_{\beta} e_{\delta}\right)=\left(a_{\gamma} a_{\beta} a_{\alpha} a_{\delta}, e_{\gamma} e_{\beta} e_{\alpha} e_{\delta}\right)
$$

(by the normality of $B$ and the commutativity of $C$ )

$$
=\left(a_{\gamma}, e_{\gamma}\right)\left(a_{\beta}, e_{\beta}\right)\left(a_{\alpha}, e_{\alpha}\right)\left(a_{\delta}, e_{\delta}\right) \text {. }
$$

Therefore, $C \infty B(\Gamma)$ is $N$-inversive.

REMARKS 1. For L.N [R.N]-inversive semigroup, we can establish an analogous result to Theorem 5 . We present it without proof.

THEOREM. A semigroup is isomorphic to the spined product of a C-inversive semigroup and a left [right] normal band if and only if $S$ is L.N [R.N]-inversive.

2. It is also true that a semigroup $S$ is isomorphic to the spined product of a weakly C-inversive semigroup and a [left, right] normal band if and only if $S$ is weakly $N[L . N, R . N]$-inversive. We also omit its proof. 
4. Classification of permutation idetities, Let $\Omega$ be the collection of all semigroups having type $T .^{1}$ Let $P_{1}=P_{2}$ and $Q_{1}=Q_{2}$ be permutation identities. If every semigroup of $\Omega$ satisfying $P_{1}=P_{2}$ satisfies $Q_{1}=Q_{2}$ and conversely every semigroup of $\Omega$ satisfying $Q_{1}=Q_{2}$ satisfies $P_{1}=P_{2}$, then $P_{1}=P_{2}$ and $Q_{1}=Q_{2}$ are said to be equivalent with respect to $\Omega$. It was shown by Kimura and the author [17] (the proof is given by [12]) that the permutation identities are classified into four distinct equivalence classes with respect to the collection of bands. That is; let $x_{1} x_{2} \cdots x_{n}=x_{p_{1}} x_{p_{2}} \cdots x_{p_{n}}$ be a permutation identity. Then, the following proposition is true with respect to the collection of bands:

$$
\left\{\begin{array}{l}
x_{1} x_{2} \cdots x_{n}=x_{p_{1}} x_{p_{2}} \cdots x_{p_{n}} \text { is equivalent to } \\
\text { (I) commutativity if } p_{1} \neq 1 \text { and } p_{n} \neq n ; \\
\text { (II) left normality if } p_{1}=1 \text { and } p_{n} \neq n ; \\
\text { (III) right normality if } p_{1} \neq 1 \text { and } p_{n}=n ; \\
\text { (IV) normality if } P_{1}=1 \text { and } p_{n}=n .
\end{array}\right.
$$

In this section, we shall show that (4.1) is also true with respect to the collection of regular semigroups.

THEOREM 6. Let $x_{1} x_{2} \cdots x_{n}=x_{p_{1}} x_{p_{2}} \cdots x_{p_{n}}$ be a permutation identity. Then the proposition (4.1) is true with respect to the collection of regular semigroups.

Proof. Suppose that a regular semigroup $S$ satisfies a permutation identity $x_{1} x_{2} \cdots x_{n}=x_{p_{1}} x_{p_{2}} \cdots x_{p_{n}}$. Since $S$ is $N$-inversive, the set $B$ of idempotents of $S$ is a band.

(I ) If $p_{1} \neq 1$ and $p_{n} \neq n$, then by the above-mentioned result of [17] the band $B$ is commutative and hence $S$ is weakly $C$-inversive. Let $x x^{\prime}=e$ and $y y^{\prime}=f$. Then, $x y=e x y f=e y x f$ (by the normality of $S)=$ efyxef $=$ feyxfe $=y x$ (by Lemma 9). Hence, $S$ satisfies commutativity.

(II) If $p_{1}=1$ and $p_{n} \neq n$, then the band $B$ is left normal and hence $S$ is weakly $L . N$-inversive. Let $x x^{\prime}=e, y y^{\prime}=f$ and $z z^{\prime}=g$. Then, $x y z=x f y z g=x f z y g$ (by the normality of $S$ ) $=x f g z y f f g=$ $x g f z y f g f$ (by the normality of $S$ and the left normality of $B$ ) $=$ $x g f z y g f=x z y$ (by Lemma 9). Hence, $S$ satisfies left normality.

(III) Similarly, in the case $p_{1} \neq 1$ and $p_{n}=n$ it is easily proved that $S$ satisfies right normality.

(Iv) In the case $p_{1}=1$ and $p_{n}=n$, it is obvious that $S$ satisfies

$1 T$ is a type of semigroup such that if one of two isomorphic semigroups has type $T$, then so also has the other. 
normality.

Conversely, it is also obvious that a regular semigroup satisfying commutativity [left normality; right normality; or normality] satisfies any permutation identity $x_{1} x_{2} \cdots x_{n}=x_{p_{1}} x_{p_{2}} \cdots x_{p_{n}}$ with $p_{1} \neq 1$ and $p_{n} \neq n\left[p_{1}=1\right.$ and $p_{n} \neq n ; p_{1} \neq 1$ and $p_{n}=n$; or $p_{1}=1$ and $p_{n}=n$ respectively].

REMARK. Since commutativity, left normality, right normality and normality are nonequivalent to each other with respect to the collection of bands, they are also nonequivalent with respect to the collection of regular semigroups.

5. Characterizations of $N[L . N, R . N, C]$-inversive semigroups. From Theorems 3 and 5, we obtain the following

CoRollary 1. For a semigroup $S$, the following conditions are equivalent:

(1) $S$ is regular and satisfies a permutation identity.

(2) $S$ is $N$-inversive.

(3) $S$ is isomorphic to the spined product of a C-inversive semigroup and a normal band.

Further, in this case $S$ is a band of groups and accordingly $S$ is both left and right regular (in the sense of [3], p. 121).

Also, we have

COROLlary 2. For a semigroup $S$, the following conditions are equivalent:

(1) $S$ is regular and satisfies left [right] normality $x y z=x z y$ $[x y z=y x z]$.

(2) $S$ is L.N [R.N]-inversive.

(3) $S$ is isomorphic to the spined product of a C-inversive semigroup and a left [right] normal band.

Proof. It is obvious that the conditions (1) and (2) are equivalent to each other. The equivalence of the conditions (2) and (3) follows from Remark 1 of Theorem 5 .

As was stated in the $\S 3$, it is easy to see that a semigroup with an identity element is commutative if it satisfies a permutation identity. Therefore, especially a group satisfying a permutation identity is commutative. Further, the following shows that an inverse semigroup satisfying a permutation identity is necessarily commutative. 
CoRollary 3. For a semigroup $S$, the following conditions are equivalent:

(1) $S$ is regular and commutative.

(2) $S$ is an inverse semigroup satisfying a permutation identity.

(3) $S$ is C-inversive.

(4) $S$ is a commutative compound semigroup of a collection of commutative groups having a semilattice as its index set. ${ }^{2}$

Proof. It is easy to see that the condition (1) implies the condition (2). Let $S$ be an inverse semigroup satisfying a permutation identity. Since $S$ is regular, it is $N$-inversive. Also, since $S$ is an inverse semigroup any two idempotents of $S$ commute. Take any elements $x, y$ of $S$, and let $x x^{\prime}=e$ and $y y^{\prime}=f$. Then $x y=e x y f=e y x f$ (by the normality of $S$ ) = efyxef $=$ feyxfe (by the commutativity of idempotents of $S)=y x\left(\right.$ since $\left.(y x)(y x)^{\prime}=f e\right)$. Hence, $S$ is commutative. Since a $C$-inversive semigroup is commutative and is a semilattice of commutative groups, it is obvious that the condition (3) implies the condition (4). Finally, it is also obvious that the condition (4) implies the condition (1).

\section{REFERENCES}

1. A. H. Clifford, Semigroups admitting relative inverses, Ann. of Math. 42 (1941), 1037-1049.

2. - Bands of semigroups, Proc. Amer. Math. Soc. 5 (1958), 499-504.

3. A. H. Clifford and G. B. Preston, Algebraic theory of semigroups, Amer. Math. Soc., Providence, Rhode Island, 1961.

4. N. Kimura, The structure of idempotent semigroups (1), Pacific J. Math. 8 (1958), $257-275$.

5. D. McLean, Idempotent semigroups. Amer, Math. Monthly 61 (1954), 110-113.

6. G. B. Preston, Inverse semigroups, J. London Math. Soc. 29 (1954), 396-403.

7. - Representations of inverse semigroups, J. London Math. Soc. 29 (1954), 411-419.

8. G. Thierrin, Demi-groupes inversés et rectangulaires, Acad. Roy. Belg. Bull. Cl. Sci. 41 (1955), 83-92.

9. V. V. Vagner, Generalized groups, Doklady Akad. Nauk. SSSR (N.S), 84 (1952), 1119-1122.

10. M. Yamada, A note on middle unitary semigroups, Kōdai Math. Sem. Rep. 7 (1955), 49-52.

11. Compositions of semigroups, Kōdai Math. Sem. Rep. 8 (1956), 107-111.

12. - The structure of separative bands, Dissertation, Univ. of Utah, 1962.

2 The concept of compound semigroup was introduced by [11]. Let $\Gamma$ be a semilattice. For each $\gamma \in I$, let $S_{\gamma}$ be a commutative semigroup. Let $S$ be the class sum of all $S_{\gamma}$ 's. Define multiplication o in $S$ such that

$(1)$ the resulting system $S(0)$ is a commutative semigroup,

$\left\{\right.$ (2) for any $\gamma \in \Gamma, S_{\gamma}$ is a subsemigroup of $S(\circ)$; $a_{\gamma} \circ b_{\gamma}=a_{\gamma} b_{\gamma}$ for any elements

$\left\{a_{\gamma}, b_{\gamma} \in S_{\gamma}\right.$, and

(3) for any $\alpha, \beta \in \Gamma, S_{\alpha} \circ S_{\beta} \subset S_{\alpha \beta}$.

In this case, $S(0)$ is called a commutative compound semigroup of $\left\{S_{\gamma}: \gamma \in \Gamma\right\}$. 
13. - Inversive semigroups. I, Proc. Japan Acad. 39 (1963), 100-103.

14. - Strictly inversive semigroups, Science Reports of Shimane Univ. 13 (1964), 128-138.

15. - Inversive semigroups. III, Proc. Japan Acad. 41 (1965), 221-224.

16. - Note on the structure of regular semigroups, Proc. Japan Acad. 42 (1966), $136-140$.

17. M. Yamada and N. Kimura, Note on idempotent semigroups. II, Proc. Japan Acad. 34 (1958), 110-112.

Received June 3, 1966. An abstract of a part of this paper has appeared in [13], [15] and [16], and also a part of $\$ 3$ of this paper was presented by the author at the Meeting of the American Mathematical Society at Stanford, April 24, 1965, under the title On regular semigroups satisfying permutation identities.

ShIMANE UNIVERSITY

MATSUe, JAPAN 


\section{PACIFIC JOURNAL OF MATHEMATICS}

\section{EDITORS}

H. SAMELSON

Stanford University

Stanford, California

J. P. JANS

University of Washington

Seattle, Washington 98105

\section{J. DugunduI}

University of Southern California Los Angeles, California 90007

RICHARD ARENS

University of California

Los Angeles, California 90024

\section{ASSOCIATE EDITORS}
E. F. BECKENBACH
B. H. NEUMANN
F. WOLF
K. YoSIDA

\section{SUPPORTING INSTITUTIONS}

\author{
UNIVERSITY OF BRITISH COLUMRIA \\ CALIFORNIA INSTITUTE OF TECHNOLOGY \\ UNIVERSITY OF CALIFORNIA \\ MONTANA STATE UNIVERSITY \\ UNIVERSITY OF NEVADA \\ NEW MEXICO STATE UNIVERSITY \\ OREGON STATE UNIVERSITY \\ UNIVERSITY OF OREGON \\ OSAKA UNIVERSITY \\ UNIVERSITY OF SOUTHERN CALIFORNIA
}

\author{
STANFORD UNIVERSITY \\ UNIVERSITY OF TOKYO \\ UNIVERSITY OF UTAH \\ WASHINGTON STATE UNIVERSITY \\ UNIVERSITY OF WASHINGTON \\ AMERICAN MATHEMATICAL SOCIETY \\ CHEVRON RESEARCH CORPORATION \\ TRW SYSTEMS \\ NAVAL ORDNANCE TEST STATION
}

Mathematical papers intended for publication in the Pacific Journal of Mathematics should be typewritten (double spaced). The first paragraph or two must be capable of being used separately as a synopsis of the entire paper. It should not contain references to the bibliography. Manuscripts may be sent to any one of the four editors. All other communications to the editors should be addressed to the managing editor, Richard Arens at the University of California, Los Angeles, California 90024 .

50 reprints per author of each article are furnished free of charge; additional copies may be obtained at cost in multiples of 50 .

The Pacific Journal of Mathematics is published monthly. Effective with Volume 16 the price per volume (3 numbers) is $\$ 8.00$; single issues, $\$ 3.00$. Special price for current issues to individual faculty members of supporting institutions and to individual members of the American Mathematical Society: $\$ 4.00$ per volume; single issues $\$ 1.50$. Back numbers are available.

Subscriptions, orders for back numbers, and changes of address should be sent to Pacific Journal of Mathematics, 103 Highland Boulevard, Berkeley 8, California.

Printed at Kokusai Bunken Insatsusha (International Academic Printing Co., Ltd.), No. 6, 2-chome, Fujimi-cho, Chiyoda-ku, Tokyo, Japan.

\section{PUBLISHED BY PACIFIC JOURNAL OF MATHEMATICS, A NON-PROFIT CORPORATION}

The Supporting Institutions listed above contribute to the cost of publication of this Journal, but they are not owners or publishers and have no responsibility for its content or policies. 


\section{Pacific Journal of Mathematics}

\section{Vol. 21, No. 2 December, 1967}

Arne P. Baartz, The measure algebra of a locally compact semigroup ..... 199

Robert F. Brown, On maps with identical fixed point sets............. 215

C. Buttin, Existence of a homotopy operator for Spencer's sequence in the analytic case ..................................... 219

Henry Werner Davis, An elementary proof that Haar measurable almost periodic functions are continuous ........................ 241

Zeev Ditzian, On asymptotic estimates for kernels of convolution transforms ...................................... 249

Robert E. Edwards, Boundedness principles and Fourier theory ......... 255

John A. Hildebrant, On compact unithetic semigroups ............... 265

Marinus A. Kaashoek and David Clark Lay, On operators whose Fredholm set is the complex plane ............................ 275

Sadao Kató, Canonical domains in several complex variables ........... 279

David Clifford Kay, The ptolemaic inequality in Hilbert geometries.... . . . 293

Joseph D. E. Konhauser, Biorthogonal polynomials suggested by the Laguerre polynomials ............................. 303

Kevin Mor McCrimmon, Macdonald's theorem with inverses .......... 315

Harry Eldon Pickett, Homomorphisms and subalgebras of multialgebras .................................... 327

Richard Dennis Sinkhorn and Paul Joseph Knopp, Concerning nonnegative matrices and doubly stochastic matrices ..............

Erling Stormer, On anti-automorphisms of von Neumann algebras ...

Miyuki Yamada, Regular semi-groups whose idempotents satisfy permutation identities .......................... 